the ways, unfortunately, is an excessively condensed presentation which, together with some inaccuracies and inadequate references to the ramifications of the various topics introduced, diminishes the value of his book. Individual chapters are heralded by thoughts from Winnie the Pooh, and there are corners of the world where these will be found harder to understand than the chapters themselves. Although workers in the field will be glad to have the latest developments available so soon, they will find reading the book very difficult, and they may well be discouraged from purchasing it at such an outrageously high price.

H. HALberstam

\section{Molecular Spectroscopy}

Molecular Spectroscopy: Modern Research. Edited by K. Narahari Rao and $\mathrm{C}$. Weldon Mathews. Pp. xiii + 422. (Academic: New York and London, May 1972.) \$25.

This volume was issued to commemorate the twenty-fifth anniversary of the Columbus Symposium on Molecular Structure and Spectroscopy, a symposium held annually in the United States at the Ohio State University. The book contains a mixture of review articles, invited talks from the 1970 symposium, and data compilations, though these three categories are not mutually exclusive. Because the material in this volume is so heterogeneous, prospective readers wishing to decide how many articles are closely allied to their own research interests have no recourse but to scan a list of contents. Such a list, with somewhat abbreviated titles, is given in the next paragraph.

One would probably classify as review articles the sections on: microwave spectroscopy (Morino and Saito), rotational levels of free radicals (Carrington), high-resolution IR of planetary atmospheres (Fox), vibrationrotation structure in asymmetric-and symmetric-tops (Mills), electronic spectroscopy of polyatomics (Innes), nuclear hyperfine structure in diatomic electronic spectra (Dunn), IR and UV matrix studies of free radicals and molecular ions (Milligan and Jacox), Fourier spectroscopy (Sanderson), grille spectrometers (Moret-Bailly), and large IR plane gratings (Rao). Written versions of 1970 invited talks include the sections on: Lamb-dip hyperfine spectrum of $I_{2}$ (Bunker), rotational energy transfer from MW line broadening (Boggs), rotational spectra of molecules with two internal degrees of freedom (Dreizler), interstellar molecules (Rank), rotational line strengths: the $\mathrm{O}_{2}^{+} \mathrm{b}^{4} \Sigma_{\mathrm{\alpha}}^{-}-\mathrm{a}^{4} \Pi_{\mathrm{u}}$ system (Zare) energy transfer research in $I_{2}$ (Steinfeld), and biprotonic phototautomerism, excimer formation, and proton tunnelling in DNA (Kasha, Horowitz, and ElBayoumi). Finally, the data compilations include: molecular IR laser emissions (Rao and Mantz), and twelve tables of physical constants, conversion factors, wavelength standards, and so on.

There is no question that each of these contributions represents, in its own way, an interesting, enlightening and useful treatment of selected subject matter by a recognized authority in the field. In my own laboratory, those who read the volume all found one or more particularly valuable articles. On the other hand, readers also found article(s) they felt might well have been omitted. Unfortunately, there was no general agreement in selecting individual articles for praise or criticism.

The most attractive general feature of the book to me is the set of excellent longer review articles with large numbers of reference citations, which will be useful for the next several years to anyone beginning work on a problem in one of the areas covered. The line lists and tabulations of constants are also convenient, though they are often accessible elsewhere in the literature.

Perhaps the most severe criticism of the book which comes to mind concerns not the individual articles themselves, but rather the collection as a whole. I am not in favour of the proliferation of random review volumes and/or conference proceedings such as this, which offend one's sense of order and complicate one's literature searches by not fitting neatly into the format of already existing periodic scientific communication channels. JON T. HougeN

\section{Oral Biology}

The Marmoset Periodontium in Health and Disease. By B. M. Levy, D. Dreisen and S. Bernick. Pp. 89. (S. Karger: Basel, London and New York, 1972.) 39 Sw. francs; $£ 4.30 ; \$ 10$.

THIS, the first volume in a series entitled Monographs in Oral Science, contains no editorial statement about the scope and aim of the series; nevertheless, the need for animal models to study prevalent dental disease such as gingivitis and pyorrhoea is sufficient reason for this topic to be chosen to initiate the series.

The marmoset, or "manikin", is susceptible to periodontal disease, especially in captivity. Its small size, near-human dentition, and ready availability have made it a most suitable subject for the intensive study of periodontal biology and the reaction of dental supporting tissues to environmental change and injury.

The monograph, in two principal parts, begins with a detailed descrip- tion of the development of deciduous and permanent dentitions of the marmoset, the structure of the adult periodontium, and its change with increasing age. The text is crisp, and the blackand-white illustrations comprehensive and clear. A monograph on comparative anatomy gains immeasurably by frequent, apposite references to the human situation. That this booklet generally fails to draw these comparisons diminishes its value as a reference work. The deficiency is remedied in the second part devoted to the initiation and extension of periodontal disease. In discussing the role of the external and internal oral environment in the ecology of the periodontium, convincing parallels are drawn between the human and the non-human primate model.

The role of bacteria, viruses, dental plaque and saliva, all key factors in the external environment, has been examined and compared with their suspected role in periodontal disease in man. Dietary changes, vitamin deficiency, and endocrine imbalance have all been employed as tools to influence the internal environment of the periodontium. Results from these valuable studies in the marmoset support Frenkel's contention that "Spontaneous models are most useful to provide inductive hypotheses concerning disease processes in man". If the contents of this slim volume are an indication of things to come, then the series will be of the utmost significance to oral biologists.

D. E. Poswillo

\section{Human Neurophysiology}

Neurophysiology Studied in Man. Edited by G. G. Somjen. (Proceedings of a Symposium held in Paris at the Faculté des Sciences, July 1972.) Pp. $x i+487$. (Excerpta Medica: Amsterdam, 1972.) Dfl. 108 ; $\$ 33.75$.

THIS symposium, with about ninety contributors, contains as its most distinctive part a great many observations relevant to normal neurophysiology that were made as incidents in treating patients, and could not have been made without the opportunities provided by therapeutic or diagnostic procedures. Papers within this field make up nearly half of the book, and cover the field so widely that the book is quite a good guide to its literature.

Another large part of the book (also nearly half) describes experiments on normal human volunteers. This field is too large for there to be any possibility of covering it in half a symposium, and papers within it look almost randomly selected. They include, I think, the best and the worst in the whole symposium.

Two or three papers are primarily about diseases, and hardly touch neurophysiology. G. S. BRINDLEY 